\title{
ANALYSIS OF FDM AND TDM FREQUENCY: THROUGH STATISTICAL TIME-DIVISION MULTIPLEXING
}

\author{
Dr. Nirmla Sharma \\ Asst. Professor, Computer Science, \\ KKU University Abha, Saudi Arabia
}

\begin{abstract}
- it has remained detected that record of separate data-communicating policies $y$ involves uncertain facts level. However, message broadcasting frequently have advanced bandwidth. By way of per result double interactive situations do not occupy the volume of a data association. Therefore multiplexing remains to clear method that permits concurrent the broadcast of numerous indications through distinct statistics association. Actual contracted group of the bright signal from dissimilar have mutual to create an extensive group of bright. The handset indications have divided through de-multiplexer.

By way of with Synchronous TDM, statistical multiplexers similarly must numerous input/ output positions through defense connected toward every of them. Throughout response, multiplexer images contribution defenses, gathering statistics awaiting structure has occupied combine structure. FDM distributes the network into numerous, then lesser rate series to put up more consumers, though TDM distributes a network through assigning a period historical for every network. TDM offers to improved elasticity associated toward Frequency Division Multiplexing.
\end{abstract}

Keywords- Analysis, Frequency, Frequency-division multiplexing and Statistical Time-division Multiplexing

\section{INTRODUCTION}

System ability can remain mutual between statistics of connecting positions objective similar huge aquatic pipeline can transfer aquatic toward distinct lines next to after. Utmost mutual practice for multiplexing is long-haul the communication by coaxial cable, microwave and optical fiber. The multiplexer has associated de-multiplexer through particular statistics association. Multiplexer associations (multiplexes) figures since these ' $n$ ' contribution positions combine communicates them finish great ability statistics association. It has multiplexed to close and transport toward production positions. Multiplexing methods can be classified interested in succeeding three kinds:

i. Frequency-division multiplexing (FDM): is current toward recycled widely now radio and TV broadcast. Rate range is separated attached in numerous rational stations to produce every consumer limited control of an individual occurrence group. ii. Time-division Multiplexing (TDM): is named synchronous TDM, which is recycles multiplexing digitized expression stream. Consumers proceed chances by complete the network used for less rupture of the period.

iii. Numerical TDM: is named asynchronous TDM, which develops scheduled competence of synchronous TDM. Units and methods have measured in feature FrequencyDivision Multiplexing (FDM) "Mohammed, H. A. (2013)".

Frequency division multiplexing is the bandwidth of particular somatic middle partitioned into numerous free rate stations. Free communication signals have interpreted into dissimilar rate bands by variation methods, which are jointed through a direct summing route in the multiplexer toward compound indication. The carriers recycled to control the discrete communication indications have named sub-carriers exposed like $\mathrm{f} 1, \mathrm{f} 2 \ldots \mathrm{fn}$. Through acceptance close indication has established of band-pass cleans which divides separate frequency stations. Group permit filter productions have demodulated then scattered to production stations.

Practice of protector groups is in FDM. If stations are actual close toward supplementary, it indications toward interchannel fractious exchange. Stations might remain divided through bands of idle the bandwidth toward stop inter-channel fractious the exchange "White, Curt. (2007)". Idle stations among every following station are recognized as protector groups as shown in figure 1 .

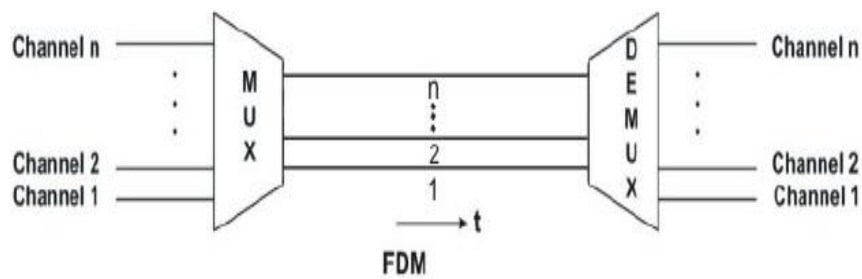

Fig1. Undeveloped WDM multiplexing \& de-multiplexing

\section{PROBLEM STATEMENT}

FDM frequency has recycled in radio transmissions and TV systems. Meanwhile, the rate group recycled used for expression broadcast now handset system is $4000 \mathrm{~Hz}$, used for 


\section{International Journal of Engineering Applied Sciences and Technology, 2019 \\ Vol. 4, Issue 4, ISSN No. 2455-2143, Pages 155-158 \\ Published Online August 2019 in IJEAST (http://www.ijeast.com)}

specific wire of $48 \mathrm{KHz}$ bandwidth, in 70 to $108 \mathrm{KHz}$ collection; twelve distinct $4 \mathrm{KHz}$ represent stations might be recycled intended for communicating twelve dissimilar communications concurrently. Every wireless device and TV location, now has assured transmission region, is selected a detail transmission rate, so that free the networks can be directed concurrently in dissimilar the transmission region. For instance, AM wireless has practiced 540 to $1600 \mathrm{KHz}$ rate groups though FM wireless practices 88 to $108 \mathrm{MHz}$ rate groups.

\section{DESIGN AND ANALYSIS OF WAVELENGTH-DIVISION MULTIPLEXING}

Wavelength-division multiplexing (WDM) is theoretically identical by way of FDM, excluding that multiplexing and demultiplexing includes bright indications communicated complete fiber-optic stations. The indication is joining and dissimilar occurrence indications. Though, the alteration is that occurrences are unlimited. It is considered to develop figures amount ability of fiber-optic wire "Harold et al. (2006)".

Multiplexing and de-multiplexing of bright indications can remain ended by sustenance of prism by way of above figure1. From the simple information of physics distinguish that light signal is determined through dissimilar volume based happening position of occurrence then wave-length of bright by way of displayed through dissimilar colors in number. Unique prism implements character of multiplexer through joining illuminations consuming dissimilar rates after foundations. The compound indication can be communicated finished an optical fiber wire ended extended spaces uncertainty necessary. Next to finish of optical fiber wire compound indication has applied toward prism to prepare the opposite process of de-multiplexer "Jing et al. (2010)".

Time-Division Multiplexing (TDM) now frequency division multiplexing, altogether indications control at the identical period through dissimilar rates, then in Time-division multiplexing all signals function by identical rate at dissimilar intervals. This is an improper band broadcast system, anywhere an electronic commentator consecutively sections altogether data basis and associations them to procedure a merged improper group indication, which actions complete the broadcasting combine existence de-multiplexed interested in suitable free communication indications through consistent commentator at the acceptance. Received statistics after every foundation have protected temporarily. Every defense is bit before single cipher now distance. Defenses have perused consecutively method to merge the statistics stream. The test process is suitably prompt consequently that every defense has deflated earlier additional statistics could attain. Compound statistics ratio need remain at least identical toward entirely of distinct statistics amounts. The compound indication could be communicated openly before finished the modem. Multiplexing process has presented in figure2. The compound signal has certain deceased space among the following tested pulses, which is critical to avoid inter-channel cross discussions "Jyotsana et al. (2013)".

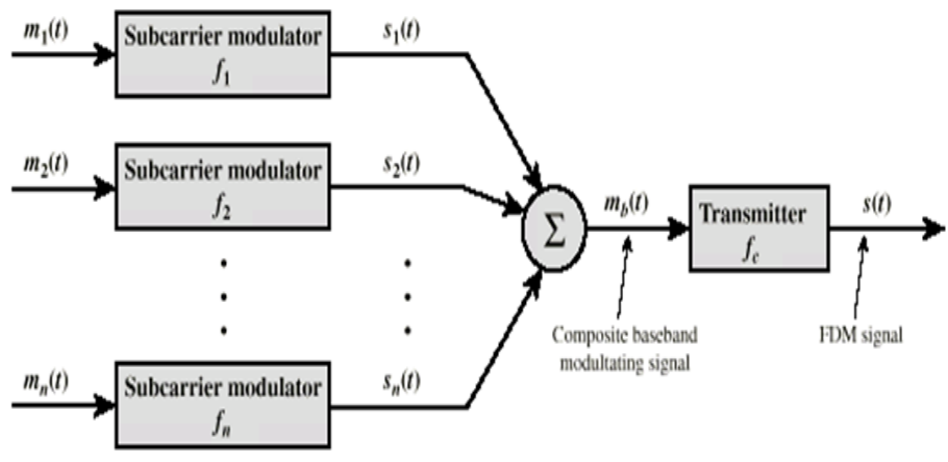

(a) Transmitter

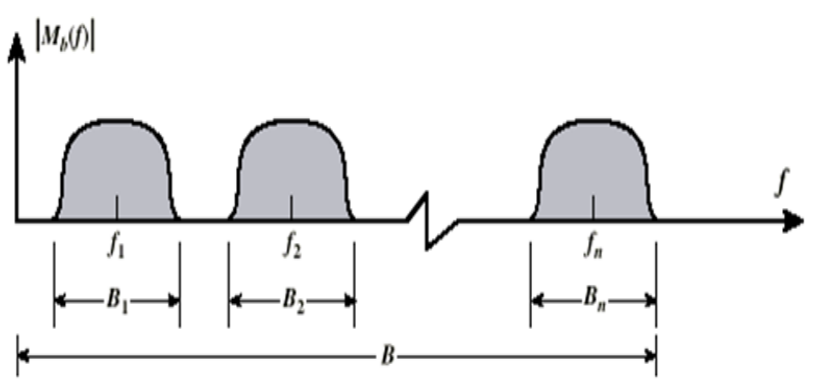

(b) Spectrum of composite baseband modulating signal

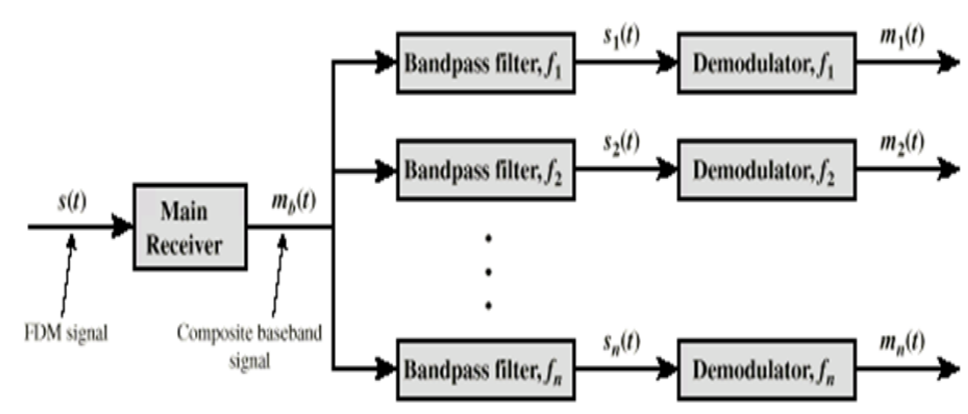

(c) Receiver

Fig2 Time division multiplexing process as exposed in the system [4]

Synchronous TDM is named synchronous mostly since every period opening has reassigned toward static foundation. Period openings have communicated unrelatedly of whether bases must several statistics toward direct or not. Later, used for moving ease to execution and station ability missed. Though static transfer has recycled TDM, strategies can switch bases of statistics degrees. This has completed through conveying rarer periods each sequence toward measured 


\section{International Journal of Engineering Applied Sciences and Technology, 2019 \\ Vol. 4, Issue 4, ISSN No. 2455-2143, Pages 155-158 \\ Published Online August 2019 in IJEAST (http://www.ijeast.com)}

contribution strategies than quicker strategies. Multiplexing and de-multiplexing action for synchronous TDM have exposed "Willner et al. (2011)".

\section{Result AND Discussion (Statistical Time- DIVISION MULTIPLEXING)}

The unique weakness of TDM method by way of deliberated previous is minimum structure misused. A capable unusual toward this synchronous TDM is statistical TDM, similarly recognized by way of asynchronous TDM or Quick TDM. It energetically assigns period slits scheduled request toward isolated contribution networks, therefore exchangeable networkability "Lange et al. (2011)".. Next to acceptance finish, de-multiplexer accepts structure and allocates figures to suitable defences. The variance among synchronous TDM and asynchronous TDM has demonstrated through the support of figure2. It might remain distinguished that slits continue unutilized in circumstance synchronous TDM, then the slits have completely developed important to reduce period for broadcast and improved operation of the bandwidth of medium. Now circumstance of statistical TDM, figures every space need have statement portion, which classifies basis of figures "Jianwei et al. (2000)". Before statistics attain after and are circulated toward input/ output positions randomly, report statistics is toward declare correct distribution as presented in figure 3 .

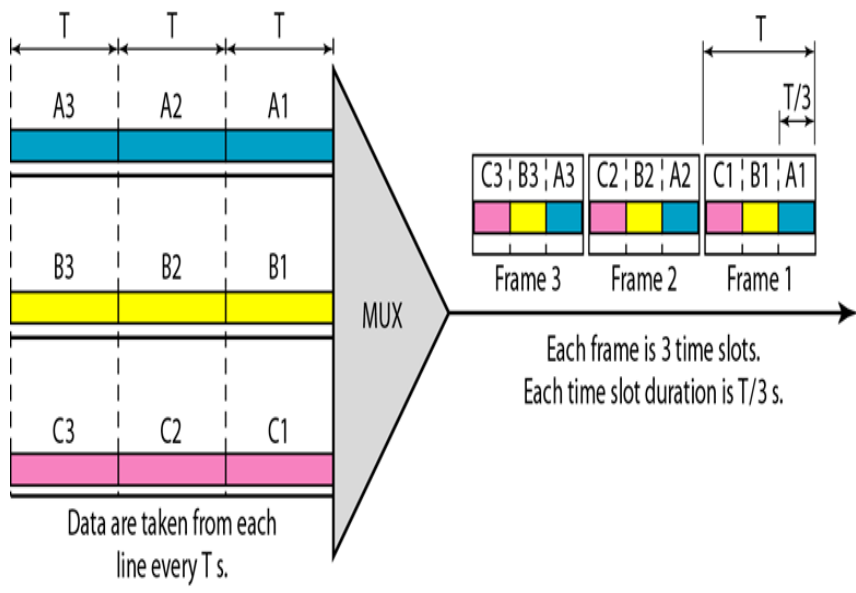

Fig3. Synchronous versus asynchronous TDM [7]

Frequency division multiplexing (FDM) has method communicates numerous indications wireless system "Singh et al. (2010)". Every moderate gives up separately at the exact occurrences. This arrangement offers the "orthogonally" in this practice, which stops the demodulators from sighted occurrences other than their individual. The simple method of FDM is demonstrated in figure4 "Enderes et al. (2000)".

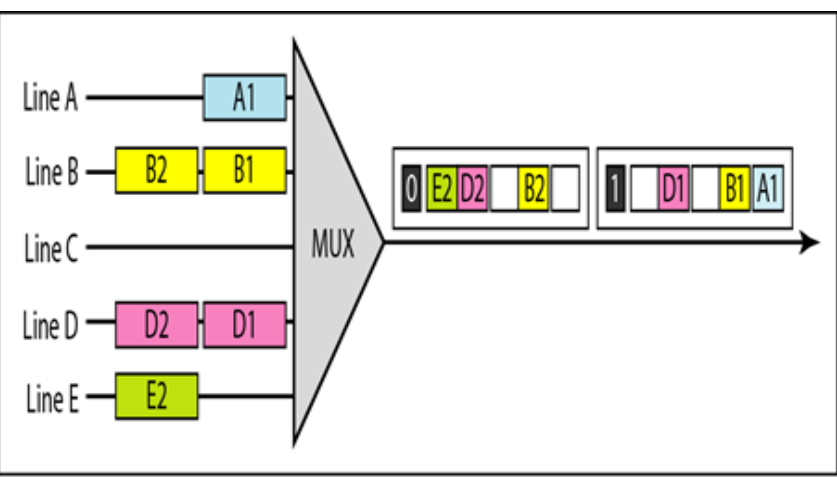

a. Synchronous TDM

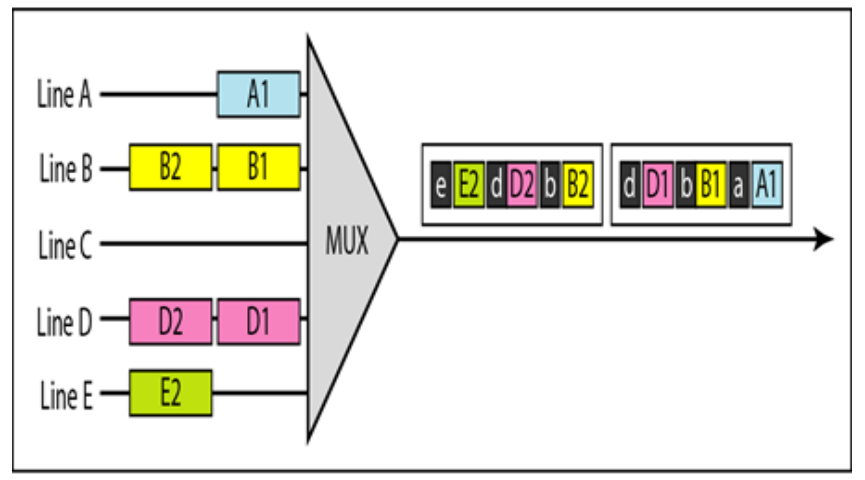

b. Statisitical TOM

Fig4. Address overhead in asynchronous TDM Statistical [8]

\section{CONCLUSION}

Frequency Division Multiplexing shows to improve latency associate toward Time Division Multiplexing. Time Division Multiplexing and Frequency Division Multiplexing can be recycled in the cycle. Beside by the tested pulses, one coordinating pulse has directed in every phase. These figures pulse beside by the switch statistics form a border. Every of these structures enclose series of period opening now every structure, unique or periods have dedicated toward every statistics foundation. Concentrated bandwidth (statistics frequency) of TDM organization must be at slightest identical toward similar statistics frequency of bases. Next to finish of optical fiber wire compound indication has applied toward additional prism to prepare the opposite process the purpose of de-multiplexer. 


\section{International Journal of Engineering Applied Sciences and Technology, 2019 \\ Vol. 4, Issue 4, ISSN No. 2455-2143, Pages 155-158 \\ Published Online August 2019 in IJEAST (http://www.ijeast.com)}

\section{FUTURE WORK}

Furthermore, once nodes run to access the system, certain capable procedures for developing the data association are identical and critical. Once the bandwidth of standard is better than indications toward remain communicated complete network, the medium can be common through than the network of indications. The procedure of creating actuals practice available the network ability is named the Multiplexing.

\section{REFERENCE}

[1] Mohammed, H. A. (2013). "Optical Time Division Multiplexing (OTDM) and Hybrid WDM/OTDM PON Performance Investigation”, ISSN, 4(3), pp. 2278-4209

[2] White, Curt. (2007). "Data Communications and Computer Networks", Boston, MA: Thomson Course Technology, pp. 140-143, ISBN 1-4188-3610-9

[3] Harold et al. (2006). "Communication Systems: Analysis and Design", Prentice Hall, ISBN 0-13-040268-0

[4] Jing et al. (2010). "Power consumption comparison between point-to-point WDM and OTDM systems, transparent Optical Networks (ICTON)", International Conference on digital object identifier, pp. 1-4

[5] Jyotsana et al. (2013). "Simulative Comparison of 40 Gbps OTDM Transmission System operating at S-band and Cband over ITU G.653", International Journal of Advanced Research in Computer and Communication Engineering, 2(8)

[6] Willner et al. (2011). "Major Accomplishments in 2010 on Optical Fiber Communication", IEEE Photonics Journal, Vol. 3, No. 2, pp. 320-324.

[7] Lange et al. (2011). "Energy Consumption of Telecommunication Networks and Related Improvement Options", Journal of Selected Topics in Quantum Electronics, 17(2)

[8] Jianwei et al. (2000). "Statistical Multiplexing over DSL Networks", Prentice Hall Publishers

[9] Enderes et al. (2000) "Impact of Statistical Multiplexing on voice quality in cellular networks", MSWIM 2000 Boston MA USA. Copyright ACM 2000 1-58113-304-9/00/08

[10] Singh et al. (2010). "Performance analysis of OTDM system in the presence of FEC using symmetric MachZehnder switch, Optik" 121 pp. 326-329 